\title{
Relação entre bullying e sintomas depressivos em estudantes do ensino fundamental
}

\author{
Associations between bullying and depressive \\ symptoms in elementary students
}

\author{
Bruna Garcia FORLIM ${ }^{1}$ \\ Ana Carina STELKO-PEREIRA ${ }^{2}$ \\ Lúcia Cavalcanti de Albuquerque WILLIAMS ${ }^{3}$
}

\begin{abstract}
Resumo
Considerável atenção da ciência vem sendo dada ao impacto negativo do bullying, contudo poucos estudos investigaram especificamente as consequências do bullying de acordo com os tipos de envolvimento (alvo, autor, alvo/autor). O presente estudo examinou a relação entre tipos de bullying e sintomas depressivos. Responderam a um questionário brasileiro sobre violência escolar (Escala de Violência Escolar - Versão Estudantes) 348 estudantes - 53,4\% do sexo feminino - do $6^{\circ}$ ao $9^{\circ}$ ano, em média com 13,3 anos ( $D P=1,3$ ). Segundo regressão logística não linear, os alunos alvos e autores de bullying apresentaram 5 vezes mais chance de ter sintomas depressivos do que os outros estudantes. Sexo, idade, ter sido reprovado, ser exclusivamente vítima não foram variáveis significativas para predizer depressão quando consideradas conjuntamente. Esses resultados são similares a pesquisas internacionais que apontaram o fato de alvos/autores (simultaneamente) de bullying terem mais problemas emocionais do que alvos e autores. Enfatizam, também, a necessidade de pesquisas futuras considerarem a especificidade dos estudantes que são alvos/autores.
\end{abstract}

Palavras-chave: Bullying; Depressão; Violência na escola.

\begin{abstract}
Considerable attention has been given by science to the negative impact of bullying, however, few studies have specifically investigated bullying consequences according to the type of bullying involvement (victim, bully, bullyl victim). The present study examined the relationship between the type of bullying involvement and depressive symptoms. A group of 348 students, 53.4\% female, from 6-9 $9^{\text {th }}$ grade, with a mean age of 13.3 years $(S D=1.3)$ answered a Brazilian School Violence Scale (Escala de Violência Escolar - Versão Estudantes). Utilizing a logistic non-linear regression analysis, bully/victim students displayed a 5 times greater chance of having depressive symptoms than the other students. Gender, age, school failure, and being exclusively a victim were not significant variables for predicting depression when considered together. These results are similar to international studies, which also indicate that bullies/victims have more emotional problems than victims and bullies per se. These findings emphasize the need to further investigate the specificity of students who are bullies/victims.
\end{abstract}

Keywords: Bullying; Depression; School violence.

$\nabla \nabla \nabla$

1 Psicóloga. São Paulo, SP, Brasil.

2 Universidade Estadual do Ceará, Coordenação de Psicologia, Programa de Pós-Graduação em Saúde Coletiva. Fortaleza, CE, Brasil.

3 Universidade Federal de São Carlos, Departamento de Psicologia, Laboratório de Análise e Prevenção da Violência. Rod. Washington Luiz, km 235, Jardim Guanabara, 13565-905, São Carlos, SP, Brasil. Correspondência para/Correspondence to: L.C.A. WILLIAMS. E-mail: <williams@ufscar.br>.

O presente estudo é parte de um estudo maior de doutorado de Psicologia de autoria de A.C. STELKO-PEREIRA, intitulado "Avaliação de um programa preventivo de violência escolar: planejamento, implantação e eficácia”, sob a orientação de L.C.A. WILLIAMS. Apoio: Fundação de Apoio à Pesquisa de São Paulo (Processo: 2008/10681-7). 
O fenômeno bullying refere-se a circunstâncias nas quais um indivíduo é exposto repetidamente por pares a ações negativas intencionais, que podem ser de natureza física, psicológica ou sexual. Geralmente, há uma desigualdade de poder entre o agressor e o alvo, que não encontra um modo eficiente para se defender (Olweus, 1993). As formas de envolvimento dos indivíduos em bullying são: autor de bullying (agressor), alvo (vítima), alvo/ autor de bullying (agressor/vítima) e testemunha (Kumpulainen et al.,1998; Sounrander, Helstelä, Helenius, \& Piha, 2000). Contudo, não se sabe exatamente se as consequências do bullying variam de acordo com o modo de envolvimento.

Alguns estudos verificaram consequências negativas de ter sido alvo de bullying, mas sem ter diferenciado da amostra quem pudesse ser tanto alvo quanto autor. Carlisle e Rofes (2007) estudaram o efeito do bullying no longo prazo, por meio de entrevistas com 15 homens adultos que voluntariamente responderam a anúncios de jornais. Os participantes atribuíram um efeito substancial e duradouro do bullying sofrido na escola, especialmente: sintomas depressivos, sentimento de vergonha, ansiedade, dificuldades de relacionamento interpessoal, principalmente amoroso, em atividades grupais e com figuras de autoridade.

Na meta-análise de Hawker e Boulton (2000), envolvendo estudos em língua inglesa, publicados no período de 1978 a 1997, notou-se relação entre ser alvo de bullying e desenvolver depressão, isolar-se, apresentar transtorno de ansiedade e autoavaliação social e global negativas. Do mesmo modo, em pesquisa com 123700 estudantes de 11 a 13 anos de idade, em 28 países, Due et al. (2005) apontaram prováveis consequências após ser alvo de bullying: a) somáticas, como dores na cabeça e no estômago, e b) psicológicas, como nervosismo, solidão e sentimento de exclusão.

Dados similares quanto a sintomas depressivos foram encontrados no estudo de Kumpulainen et al. (1998), com 5813 alunos do ensino fundamental finlandês. Alvos de bullying dos dois sexos tiveram escores altos para: a) anedonia, que se 368 atividades antes prazerosas, b) autoestima baixa e c) humor deprimido. Comportamentos externalizantes e hiperatividade foram especialmente relatados em alvo/autores de bullying, sendo tal grupo mais frequentemente encaminhado a consultas psiquiátricas.

Do mesmo modo, Fleming e Jacobsen (2009) avaliaram a relação entre bullying e sintomas depressivos em 8131 estudantes chilenos de 13 a 15 anos. Os resultados indicaram que estudantes que reportaram sofrer bullying no mês anterior eram mais prováveis de apresentar sintomas depressivos e não ter amigos quando comparados com os que não sofriam bullying. Adicionalmente, tinham mais chance de apresentar comportamentos prejudiciais à saúde, como fazer uso de tabaco, álcool ou drogas ilícitas, de cabular aulas e de se envolver em brigas no ano anterior.

Outros estudos, além de verificar as consequências negativas em ser alvo de bullying, também verificaram as consequências de ser autor ou autor/ alvo. Sourander et al. (2000) investigaram 898 crianças finlandesas quando tinham 8 anos e, posteriormente, quando estavam com 16 anos. Perceberam que o fato de ter tido problemas emocionais e comportamentais aos 8 anos foi associado à autoria de bullying, bem como ser alvo 8 anos depois. Autorrelatos que indicavam altos níveis de sintomas depressivos (baixa autoestima, imaturidade, solidão, baixa habilidade de resolução de problemas) foram correlacionados, 8 anos depois, tanto com autoria quanto com ser alvo de bullying.

Fekkes, Pijpers e Verloove-Vanhorick (2004) encontraram uma associação consistente entre sofrer bullying e queixas de saúde em 2766 crianças, de 9 a 12 anos da Holanda. Entretanto, a relação entre ser autor de bullying e apresentar queixas de saúde e depressão não foi encontrada. Porém, houve relação entre queixas de saúde, depressão e ser autor/alvo. Os alunos autor/alvo tiveram maior risco para dor abdominal, problemas de apetite, enurese, cansaço e sentir-se infelizes.

Kaltiala-Heino, Rimpelä, Marttunen, Rimpelä e Rantanen (1999) avaliaram a relação entre bullying, depressão e ideação suicida em 16410 estudantes de 14 a 16 anos da Finlândia. A 
depressão ocorreu com maior frequência para os alunos que relataram ser tanto autor como alvo de bullying. Controlando as variáveis idade e sexo, os pesquisadores encontraram maior risco de sofrer depressão para os alunos alvo/autor de bullying (Odds Ratio-OR=9.4), seguidos pelas vítimas $(\mathrm{OR}=5,1)$ e autores $(\mathrm{OR}=4,5)$. Além disso, para os meninos, a ideação suicida foi associada somente à autoria de bullying .

Perren, Dooley, Shaw e Cross (2010) pesquisaram a associação entre ser alvo, autor e alvo/ autor de bullying e sintomas depressivos em 374 e 1320 estudantes da Suíça e da Austrália respectivamente. O grupo que mais apresentou sintomas depressivos foi o de alvo/autor de bullying, seguido pelo alvo, autor e não envolvidos.

No contexto brasileiro, nota-se uma menor atenção em estudos quanto às consequências do bullying. Em busca, feita no banco de dados Scientific Electronic Library Online (SciELO) em 2009 com a palavra-chave "bullying", não foram encontrados artigos que abordavam o tema com dados empíricos. Os demais estudos abordavam o tema com intuito exploratório, descrevendo-o ou citando-o como problema a ser estudado.

No banco de teses e dissertações da Coordenação de Aperfeiçoamento de Pessoal de Nível Superior (Capes), em novembro de 2010, utilizando-se como palavra-chave "bullying", foram encontrados 48 estudos, mas apenas quatro mencionaram as consequências do bullying com dados empíricos (Catini, 2004; Cunha, 2009; Machida, 2007). Os demais estudos concentraram-se em discorrer a respeito de outros temas referentes ao fenômeno bullying (prevalência, o papel e as concepções dos professores e gestores diante do bullying, entre outros).

Cunha (2009) estudou a relação entre sinais de depressão e agressão entre pares em 849 estudantes com média de 14,3 anos. Os dados revelaram uma correlação positiva e significativa entre o escore de depressão e as médias de todas as dimensões de agressão e vitimização avaliadas, tais como agressão direta, relacional, indireta e de vitimização. No entanto, o autor não faz distinção entre alvo, autor e alvo/autor de bullying com de- pressão. Machida (2007) investigou o relato de três mulheres e dois homens adultos vítimas de bullying no Rio Grande do Sul, analisando suas narrativas. Nessa perspectiva, as vítimas retrataram sofrimento, angústia e sentimento de impotência diante do bullying. Catini (2004) investigou o bullying em uma escola pública da periferia de Campinas (SP), a partir de desenhos e relatos de 41 alunos, que indicaram sentimento de impotência por parte dos espectadores, ansiedade e insegurança devido à expectativa de novas agressões, sentimento de revolta, impotência, culpa, e reflexos negativos na autoestima.

Pode-se observar que, apesar de existirem pesquisas que abordam a temática bullying no Brasil, poucos estudos investigaram especificamente suas consequências, contrastando-as com relação às várias formas de envolvimento (alvo, autor, alvo/ autor).

O presente estudo investigou as relações entre tipos de envolvimento em bullying (autor, alvo, autor/alvo) e sintomas depressivos em alunos, considerando a interação com as variáveis: sexo, idade e ter ou não sido reprovado.

\section{Método}

\section{Participantes}

Participaram 348 alunos que preencheram todos os itens dos instrumentos, provenientes da $6^{\mathrm{a}}$ a $9^{\mathrm{a}}$ série de duas escolas públicas de São Carlos (SP), localizadas em bairro cujo Índice Paulista de Vulnerabilidade Social era igual a 6, o que indica muito alta vulnerabilidade social (Fundação Sistema Estadual de Análise de Dados, 2002).

\section{Instrumentos}

Escala de Violência Escolar - Versão Estudantes (EVE): inicialmente denominado Questionário de Investigação de Prevalência de Violência Escolar (QIPVE), foi composto de modo a verificar a violência física, a violência psicológica/emocional e 
a violência contra o patrimônio em escolas. Com 51 questões em escala Likert, os alunos devem escolher uma alternativa (nenhuma, 1 ou 2 vezes, 3 ou 4 vezes, 5 ou 6 vezes e 7 vezes ou mais). Na avaliação de consistência interna (Stelko-Pereira, Williams, \& Freitas, 2010), o instrumento obteve um $\alpha=0,95$. O EVE resulta em uma aprimoração do QIPVE, após reformulações gráficas.

Não há critérios universais para estabelecer a pontuação mínima para que o aluno seja considerado alvo, autor e alvo/autor, pois falta normatização nesse sentido. No Brasil, além de serem poucos os instrumentos com o mesmo objetivo, eles não foram normatizados. Assim, na presente pesquisa, utilizaram-se os seguintes critérios: a) para o aluno ser considerado autor exclusivo, ele deveria ter obtido escore maior ou igual ao escore médio de autoria de violência da população investigada e escore menor do que o escore médio para vitimização; b) para o aluno ser considerado alvo exclusivo, ele deveria ter tido pontuação maior ou igual à pontuação média de vitimização e ter tido pontuação menor do que o escore médio de autoria; c) para o aluno ser considerado alvo/autor, ele deveria ter tido pontuação acima ou igual ao escore médio com relação à vitimização e com relação à autoria. Tais critérios foram exigentes, pois a análise das respostas dos alunos indicou que as variáveis vitimização e autoria apresentam curva de distribuição acentuada à direita, de modo que se o ponto de corte para vitimização e autoria fosse a moda ou a mediana, mais alunos seriam considerados alvos, autores e alvos/autores.

Inventário de Depressão Infantil (CDI): instrumento criado e validado nos Estados Unidos por Kovacs (1992), a partir de uma adaptação do Beck Depression Inventory (BDI) para adultos, foi validado para a população brasileira por Gouveia, Barbosa, Almeida e Gaião (1995). O objetivo do CDI é verificar a presença e a severidade de sintomas de depressão em crianças de 7 a 17 anos, a partir de autorrelato. $O$ instrumento é composto por 20 itens, tendo cada item 3 opções de resposta. 0 ponto de corte empregado no instrumento foi de 17 pontos conforme sugerido por Gouveia. Estudos de consistência interna indicam um alfa de 370 Cronbach entre 0,72 e 0,89 (Gouveia et al., 1995).
O projeto foi aprovado pelo Comitê de Ética em Pesquisa da Universidade Federal de São Carlos (Processo no 23112.001546.2009/57). Apenas participaram da pesquisa alunos cujos pais tivessem autorizado a participação do filho por meio do Termo de Consentimento Livre e Esclarecido (TCLE). Foram entregues brindes (canetas e doces) aos alunos que trouxessem o TCLE assinado, mesmo que o responsável tivesse recusado a participação do filho. Os alunos que apresentaram escore compatível com depressão participaram de uma entrevista psicológica individual e foram encaminhados, conforme a pertinência, à psicoterapia e/ou à avaliação psiquiátrica, entre outros.

Os instrumentos foram aplicados em sala de aula de cada série, durante cerca de 30 minutos: em primeiro lugar, foi aplicado o EVE, depois, o CDI.

Foi utilizada a técnica de regressão não linear logística (Hosmer \& Lemeshow, 1989), e a variável resposta qualitativa podia ter dois resultados possíveis: 1 (o aluno apresenta depressão) e 0 (o aluno não apresenta depressão). As variáveis preditoras consideradas na explicação da variável resposta foram: sexo, idade, se o aluno foi reprovado ou não e se o aluno era alvo, autor ou autor/alvo de bullying. As variáveis foram inseridas uma a uma e o desvio foi calculado para todas as combinações possíveis entre as cinco variáveis. Usou-se o procedimento de regressão stepwise, assim como efeitos de curvatura e de interação. Para cada modelo ajustado, foi calculado o seu desvio e o desvio parcial, de modo a testar se determinada(s) variável(eis) explanatória(s) pode(m) ser retirada(s) do modelo. O estudo do desvio parcial foi realizado para todas as combinações possíveis de variáveis.

\section{Resultados e Discussão}

As idades variaram de 13 a 18 anos; a média de idade foi de 13,3 (DP= 1,3); a maioria dos participantes era do sexo feminino $(53,4 \%)$, não havia sido reprovada $(74,0 \%)$ e apenas $6,6 \%$ tiveram escore indicativo de depressão. Em relação à prevalência de depressão, observou-se que a taxa encontrada no atual estudo está próxima aos valores de outros estudos brasileiros, como os de Cunha (2009), de 6,5\%, e Reppold e Hutz (2003), de 5,7\%. 
Apesar de não ter sido o objetivo desse estudo, parece que residir em um bairro com baixo status socioeconômico não aumentaria a prevalência de depressão em alunos brasileiros. A literatura a esse respeito apresenta dados contraditórios (Twenge \& Nolen-Hoeksema, 2002). Segundo a meta-análise de Lorant et al. (2003), de estudos desde 1979, em inglês, francês, alemão e espanhol, que investigaram a população adulta sem se ater a populações clínicas, baixo status socioeconômico está relacionado à depressão. Em contraste, a pesquisa de Twenge e Nolen-Hoeksema (2002), específica ao público infantil, investigou estudos que empregaram o CDI (mesmo instrumento deste estudo), realizando análises com informações de 61424 crianças, não sendo encontrada diferença significativa em relação a índices de depressão e status socioeconômico. Seria interessante que estudos futuros brasileiros investigassem o tema com maior profundidade.

Com relação aos $27 \%$ dos alunos que afirmaram já ter sido reprovado alguma vez, tal índice foi mais alto quando comparado a dados anuais do Instituto Nacional de Estudos e Pesquisas Educacionais Anísio Teixeira (2011): em 2009, 5\% foi reprovado. Porém, se forem considerados dados de defasagem entre idade e série escolar, tal taxa é mais baixa do que a do Instituto Brasileiro de Geografia e Estatística (2002): para a idade de 13 anos, cerca de $41 \%$ não se encontram na série esperada no estado de São Paulo. Tais diferenças podem ser explicadas: a) pelo fato de a fonte de coleta de dados ter se apoiado exclusivamente no relato dos alunos; b) por ter sido perguntado se haviam sido reprovados ao longo da sua carreira escolar e não especificamente no ano anterior; e c) pelo fato de os participantes serem alunos que estudam em escolas localizadas em áreas de baixo status socioeconômico.

A pontuação média de ser alvo de bullying foi de 14,78, e de autoria, 3,99. Cabe lembrar que o escore máximo possível para alvo de bullying era de 76, e para autoria de bullying, de 52. Seria interessante aplicar o mesmo instrumento em outros estudos, comparando-se os escores médios em diferentes populações. Sobre a prevalência de bullying, notou-se que $16 \%$ foram alvos exclusivos, $23 \%$ foram alvos/autores e $11 \%$ foram autores exclusivos.

Lopes Neto e Saavedra (2003) perceberam que $16,9 \%$ eram somente alvo, 10,9\% eram alunos que ora sofrem e ora cometem atos de bullying, $12,7 \%$ eram autores e $57,5 \%$ testemunhas, tendo sido definido o que é bullying aos participantes e depois utilizado como critério as respostas às questões: "nos últimos 3 meses, você foi vítima de bullying?" e "nos últimos 3 meses, você foi autor de bullying?". Pinheiro e Williams (2009) encontraram valores acima de 20,0\% para ser alvo, 21,0\% para alvo/autor, e 3,0\% para autor, tendo estabelecido como critério estar envolvido com bullying, ter sofrido ou cometido algum ato de bullying no mínimo 2 ou 3 vezes no mês. Já Cunha (2009) encontrou $21,0 \%$ de vítimas, 5,4\% de alvo/autor e $39,4 \%$ de autor, sendo utilizada análise de agrupamento por método hierárquico de Ward para a composição desses grupos.

Tais diferenças entre resultados de pesquisas são comuns em estudos sobre vitimização entre pares, não sendo exclusividade do Brasil, seja por diferença entre as amostras, seja pelo critério instituído para afirmar o envolvimento ou não com bullying (Pinheiro \& Williams, 2009). Alguns estudos internacionais, por exemplo, utilizam listas de comportamento, enquanto outros apresentam uma definição fechada de bullying na introdução, solicitando ao participante definir sua forma de envolvimento (Hamburger, Basile, \& Vivolo, 2011). As diferenças refletem a necessidade de se buscar maior acordo entre pesquisadores e a sistematização de instrumentos com propriedades psicométricas avaliadas e de reconhecida reputação.

Ao se investigar a relação entre depressão e as variáveis - sexo, idade, reprovação, se o adolescente é alvo de bullying e autor de bullying -, observou-se que as variáveis significativas associadas à depressão foram reprovação e ser alvo/autor de bullying (Tabela 1). Porém, cabe notar que não houve casos de autores de bullying sofrendo depressão, não sendo possível realizar cálculos de OR para essa categoria.

O aluno que foi reprovado na série escolar aumentou em 0,34 a chance de apresentar de- 
Tabela 1

Resultados para sexo, reprovação, idade e tipo de envolvimento em bullying predizendo depressão $(N=348)$

\begin{tabular}{|c|c|c|c|c|c|}
\hline & $\mathrm{n}$ & Com depressão (\%) & Sem depressão (\%) & Odds ratio relativo & $p$ \\
\hline \multicolumn{6}{|l|}{ Sexo } \\
\hline Masculino & 155 & 2 & 43 & 2,06 & \multirow[t]{2}{*}{0,14} \\
\hline Feminino & 193 & 5 & 50 & {$[0,79-5,38]$} & \\
\hline \multicolumn{6}{|l|}{ Reprovação } \\
\hline $\operatorname{sim}$ & 89 & 3 & 23 & 0,34 & \multirow[t]{2}{*}{$0,03^{*}$} \\
\hline Não & 259 & 4 & 70 & {$[0,13-0,94]$} & \\
\hline \multicolumn{6}{|c|}{ Autor exclusivo } \\
\hline Sim & 37 & 0 & 7 & - & \multirow[t]{2}{*}{-} \\
\hline Não & 311 & 11 & 82 & & \\
\hline \multicolumn{6}{|c|}{ Alvo exclusivo } \\
\hline Sim & 55 & 1 & 15 & 1,12 & \multirow[t]{2}{*}{0,87} \\
\hline Não & 293 & 6 & 78 & {$[0,27-4,58]$} & \\
\hline \multicolumn{6}{|l|}{ Autorlalvo } \\
\hline Sim & 80 & 4 & 20 & 3,7 & \multirow[t]{2}{*}{$0,01^{*}$} \\
\hline Não & 268 & 3 & 73 & {$[1,34-10,19]$} & \\
\hline \multicolumn{6}{|c|}{ Idade (anos) } \\
\hline 11 & 38 & 1 & 10 & \multirow{7}{*}{$\begin{array}{c}0,74 \\
{[0,52-1,05]}\end{array}$} & \multirow{7}{*}{0,09} \\
\hline 12 & 54 & 1 & 14 & & \\
\hline 13 & 95 & 2 & 26 & & \\
\hline 14 & 91 & 2 & 24 & & \\
\hline 15 & 50 & 1 & 14 & & \\
\hline 16 & 13 & 0 & 4 & & \\
\hline 17 & 5 & 0 & 1 & & \\
\hline
\end{tabular}

Nota: ${ }^{*} p<0,05$.

pressão em comparação com o aluno que não foi reprovado. Pode-se inferir que o aluno que foi reprovado passa a ser criticado por familiares, educadores e pares, de modo que se percebe como incapaz ou inferior. Jimerson (2001) aponta que o aluno que é reprovado passa a ter baixa autoestima e autoconfiança, assim como sentimento de impotência, tendo maior chance de desenvolver depressão. Contudo, também se sabe que o aluno com depressão tem menor disponibilidade para engajar-se nas atividades escolares, para aplicar estratégias de aprendizagem eficazes e, assim, acaba tendo menor rendimento acadêmico (Cruvinel \& Boruchovitch, 2004).

$O$ aluno que é alvo/autor de bullying aumentou em 3,7 vezes no presente estudo a chance de ter depressão, resultado semelhante a pesquisas internacionais. Fekkes et al. (2004) notaram que alvos/autores apresentaram depressão e muitas

372 queixas de saúde: tal grupo tem mais queixas somá- ticas do que as vítimas. Kaltiala-Heino et al. (1999) também notaram que o maior risco de sofrer depressão seria para o grupo de alvos/autores. 0 resultado no estudo de Perren et al. (2010) também foi semelhante ao presente estudo: houve uma maior associação entre depressão e ser alvo/autor do que com outras formas de envolvimento em bullying.

O fato de apenas ter-se encontrado relação entre ser alvo/autor e depressão e não outras formas de envolvimento, como ser vítima, entra em contraste com a literatura. Pesquisas apontaram relação entre ser vítima e ter depressão (Hawker \& Boulton, 2000; Kumpulainen et al., 1998). O presente estudo pode não ter percebido relação entre ser alvo exclusivo e depressão pelo fato de a amostra de alunos somente alvos ter sido relativamente pequena; pelo fato de o bullying na população estudada se configurar de maneira diferente de outras populações; e/ou por se tratar de alunos morando em uma 
região de alta vulnerabilidade social. Replicações desse estudo com um número maior de participantes e em escolas que atendem um público com status socioeconômico mais favorecido seriam relevantes.

O presente estudo reforça a necessidade de se investigarem as consequências do bullying e se fazerem análises separadas para alvos/autores, ou seja, empregando tanto instrumentos de vitimização quanto de autoria. Desse modo, nas pesquisas de Carlisle e Rofes (2007), Due et al. (2005), Kumpulainen et al. (1998) e Fleming \& Jacobsen (2009), apenas empregou-se instrumento que avalia se houve vitimização, de modo que, nas análises de dados, a categoria de vítimas é composta tanto pelos que são exclusivamente vítimas quanto pelos que são alvos/autores. Assim sendo, poder-se-ia superestimar as consequências para a vítima e minimizar o fato de que existe um grupo de alunos que ora é vítima, ora é autor.

Com relação ao sexo e à idade não terem sido variáveis de importância para a depressão na população estudada, percebeu-se semelhança com relação ao estudo de Twenge e Nolen-Hoeksema (2002). Segundo esses autores, não houve diferença entre sexo com relação à depressão dos 8 aos 12 anos de idade. A partir dos 13 anos, as garotas apresentam mais sintomas depressivos, porém, apesar de significativa, a diferença foi pequena $(d=0,2)$. Twenge e Nolen-Hoeksema (2002) também perceberam que os índices de depressão se mantiveram relativamente estáveis em garotos dos 7 aos 16 anos, enquanto para as garotas houve um pequeno aumento conforme envelhecem. Apesar de no presente estudo não ter sido calculado o OR entre depressão e idade para o grupo de meninos e para o grupo de meninas separadamente, notou-se que a diferença de idade não parece ser uma variável importante com relação à depressão no período da infância e pré-adolescência.

Após analisada a relação entre depressão com cada variável isoladamente, buscou-se verificar o melhor modelo para predizer depressão, considerando-se as variáveis conjuntamente. Considerando-se um nível de $5 \%$ de significância estatística, percebeu-se que apenas a variável ser alvo/ autor é de importância para o modelo, sendo o melhor modelo: Logit Depressão $=-3,2504+$ $1,6106^{*}$ Alvo/Autor, o qual expressaria a seguinte probabilidade do indivíduo ter depressão, sabendo que ele é alvo/autor de bullying:

$$
P(X)=\frac{1}{1+e^{-\left(-3,2504+1,6106^{*} \text { alv_autor }\right)}}=0,8375
$$

Desse modo, a chance de se ter depressão sendo alvo/autor de bullying é de $83,75 \%$, e o OR de ser alvo/autor ajustado para todas as variáveis do modelo seria de 5,00, ou seja, maior do que quando as variáveis foram relacionadas separadamente com a depressão. Portanto, o aluno que é alvo/autor apresenta 5 vezes mais chances de ter depressão do que o aluno que não é alvo/autor.

Assim, não se pode simplificar o fenômeno bullying em termos de alvos versus autores, pois existe um grupo que ora é autor, ora é alvo, e que merece especial atenção. Haynie et al. (2001) também encontraram que o grupo de alunos alvos/ autores tiveram significativamente piores escores do que os alunos alvos, alunos autores e alunos não envolvidos no bullying para diversas variáveis, tais como problemas de comportamento, porte de armas, má conduta, como mentir aos pais, falta de autocontrole, influência de pares desviantes e sintomas depressivos.

É importante entender quais as variáveis que prediriam ser alvo/autor. Assim, o presente estudo realizou análises a fim de verificar as variáveis que melhor explicariam ser alvo/autor. O melhor modelo ajustado para esse conjunto de dados, considerando-se um nível de significância estatística de $5 \%$, foi: Logit Alvo/Autor $=5,310+1,3663^{*}$ Depressão - 0,6306*Reprovação - 0,4209*Idade, de modo que tal função logística expressa a seguinte probabilidade:

$$
P(X)=\frac{1}{1+e^{-(5,31+1,3663 \text { depressão-0,6306reprovaçăo-0,4209idade })}}
$$

Desse modo, percebeu-se que a variável ter depressão foi a única que influenciou positivamente o ser alvo/autor, ao passo que ser reprovado e aumentar a idade influenciam negativamente. Ou 
seja, o aluno que tinha depressão seria mais provável de ser alvo/autor de bullying do que o aluno não depressivo. Ademais, conforme o aluno envelhece, a probabilidade de ser alvo/autor de bullying diminui. O OR ajustado para todas as variáveis do modelo indicou que o OR para o aluno que tem depressão é 3,92, ou seja, a cada ano de idade do aluno, há uma chance 3,92 vezes maior de quem tem depressão ser alvo/autor de bullying nessa população. No caso da variável reprovação, O OR foi de 0,53 , controlando-se a idade e ter ou não depressão. Assim, quando o aluno foi reprovado, houve uma redução de 47,00\% na chance de ser alvo/autor de bullying nessa população. Por fim, o OR para cada ano de incremento na idade foi de 0,65: a cada ano de idade do aluno, há uma redução de $34,35 \%$ no risco de ser alvo/autor de bullying.

Portanto, a partir desse modelo, considerando que o aluno tenha depressão e tenha sido reprovado, se tiver 13 anos, a probabilidade de que seja alvo/autor de bullying é de $64 \%$; se tiver 14 anos, é de 54\%; se tiver 15 anos, de $43 \%$; se tiver 16 anos, de $33 \%$; se tiver 17 anos, de $25 \%$, e se tiver 18 anos, de $18 \%$. Assim, nota-se uma tendência linear (coeficiente de Pearson $r=-0.997$ ) de decréscimo na probabilidade de ser alvo/autor de bullying à medida que a idade do aluno aumenta.

A fim de entender o relacionamento entre tais variáveis, hipóteses podem ser levantadas. Sabe-se que, em geral, alunos com comportamentos agressivos (autores e alvo/autores) tendem a perceber as ações dos outros como hostis, a aceitar a resolução de problemas de modo violento, a ter comportamentos impulsivos agressivos e dificuldade em regular suas emoções (O'Brennan, 2009). Contudo, o aluno autor/alvo nem sempre seria capaz de avaliar acuradamente quando o oponente irá se submeter a ele, de modo que acaba recebendo retaliações, tornando-se vítima. Porém, conforme aumenta a idade, o aluno teria tido mais experiências e maior desenvolvimento cognitivo, especialmente de área cerebral pré-frontal, a qual está relacionada ao controle de impulsos (Giedd, 2004), de modo que conseguiria avaliar melhor o contexto social, evitando situações em que seria alvo ou autor de bullying.
Outra hipótese é a de que, ao ser reprovado, o aluno alvo/autor pode ter mais força física e popularidade, por ser mais velho e mais experiente, do que seus colegas de classe, de modo que deixaria de ser alvo, passando a ser somente autor de bullying. Finalmente, há de se considerar, também, que sintomas depressivos podem fazer com que o aluno tenha menores condições de se defender, por ter uma postura triste e apática. Ao mesmo tempo, a depressão na adolescência também se relaciona à baixa tolerância à frustração, a comportamentos impulsivos e rompantes de agressividade, o que torna o aluno com depressão mais propenso a ser alvo/autor. Tais hipóteses poderiam ser investigadas em estudos futuros, bem como seria importante verificar se os resultados se mantêm com uma amostra maior de participantes e diferenciada quanto a status socioeconômico principalmente em relação a alunos vítimas e alunos autores.

O objetivo do presente estudo foi investigar as relações entre ser autor de bullying, alvo de bullying, autor/alvo de bullying e apresentar sintomas depressivos em crianças e adolescentes, considerando as variáveis: sexo, idade e ter sido ou não reprovado. As variáveis encontradas relacionadas à depressão foram: ter sido reprovado na escola e ser autor/alvo de bullying. Buscando-se, então, entender quais seriam as variáveis de importância para predizer ser alvo/autor, notou-se que ter depressão se relaciona positivamente, e o aumento de idade e ter sido reprovado, negativamente. Futuros estudos poderiam aprofundar essas relações, com amostras mais amplas e heterogêneas.

\section{Referências}

Carlisle, N., \& Rofes, E. (2007). School bullying: Do adult survivors perceive long-term effects? Traumatology, 13(1), 16-26.

Catini, N. (2004). Problematizando o bullying para a realidade brasileira (Tese de doutorado não-publicada). Pontifícia Universidade Católica de Campinas.

Cunha, J. M. (2009). Violência interpessoal em escolas no Brasil: características e correlatos (Dissertação de mestrado não-publicada). Universidade Federal do Paraná, Curitiba.

Cruvinel, M., \& Boruchovitch, E. (2004). Sintomas depressivos, estratégias de aprendizagem e rendimento es- 
colar de alunos do ensino fundamental. Psicologia em Estudo, 9(3), 369-378.

Due, P., Holstein, B. E., Lynch, J., Diderichsen, F., Gabhain, S. N., \& Scheidt, P. (2005). Bullying and symptoms among school-aged children: International comparative cross sectional study in 28 countries. European Journal of Public Health, 15(2), 128-132.

Fekkes, M., Pijpers, F. I., \& Verloove-Vanhorick, S. P. (2004). Bullying behaviour and associations with psychosomatic complaints and depression in victims. The Journal of Pediatrics, 144(4), 17-22.

Fleming, L. C., \& Jacobsen, K. J. (2009). Bullying and symptoms of depression in Chilean middle school students. Journal of School Health, 79(3), 130-137.

Fundação Sistema Estadual de Análise de Dados. (2002). Índice paulista de vulnerabilidade social. Recuperado em setembro 22, 2011, de http://www.seade.gov.br/ projetos/ipvs/municipios_pdf.php?letra=S

Giedd, J. N. (2004). Structural magnetic resonance imaging of the adolescent brain. Annals of the New York Academy of Sciences, 1021, 77-85.

Gouveia, V. V., Barbosa, G. A., Almeida, H. J. F., \& Gaião, A. A. (1995). Inventário de Depressão Infantil - CDI: estudo de adaptação com escolares de João Pessoa. Jornal Brasileiro de Psiquiatria, 44(7), 345-349.

Hamburger, M. E., Basile, K. C., \& Vivolo, A. M. (2011). Measuring bullying victimization, perpetration, and bystander experiences: A compendium of assessment tools. Georgia: Centers for Disease Control and Prevention.

Haynie, D. L., Nansel, T., Eitel, P., Crump, A. D., Saylor, K., Yu, K., \& Simons-Morton, B. (2001). Bullies, victims, and bully/victims: Distinct groups of at-risk youth. Journal of Early Adolescence, 21(1), 29-49.

Hawker, D., \& Boulton, M. (2000). Twenty years' research on peer victimization and psychosocial maladjustment: A meta-analytic review of cross-sectional studies. Journal of Child Psychology \& Psychiatry \& Allied Disciplines, 41(4), 441-455.

Hosmer, D. W., \& Lemeshow, S. (1989). Applied logistic regression. New York: John Wiley.

Instituto Brasileiro de Geografia e Estatística. (2002). Síntese de Indicadores Sociais 2002. Rio de Janeiro: IBGE.

Instituto Nacional de Estudos e Pesquisas Educacionais Anísio Teixeira. (2011, novembro). Data Escola Brasil. [índice de reprovação]. Recuperado em novembro 27, 2011, de http://www.dataescolabrasil.inep.gov.br/ dataEscolaBrasil/home.seam

Jimerson, S. R. (2001). Meta-analysis of grade retention research: Implications for practice in the $21^{\text {st }}$ century. School Psychology Review, 30(3), 420-437.

Kaltiala-Heino, R., Rimpelä, M., Marttunen, M., Rimpelä, A., \& Rantanen, P. (1999). Bullying, depression, and suicidal ideation in Finnish adolescents: School survey. BMJ, 319, 348-351.

Kovacs, M. (1992). Children's depression inventory manual. Los Angeles: Western Psychological Services.

Kumpulainen, K., Räsänen, E., Henttonen, I., Almqvist, F., Kresanov, K., Linna, S. L., ... Tamminen, T. (1998). Bullying and psychiatric symptoms among elementary school-age children. Child Abuse \& Neglect, 22(7), 705-717.

Lopes Neto, A. A.; \& Saavedra, L. H. (2003). Diga não para o bullying: programa de redução do comportamento agressivo entre estudantes. Rio de Janeiro: ABRAPIA.

Lorant, V., Deliège, D., Eaton, W., Robert, A., Philippot, P., \& Ansseau, M. (2003). Socioeconomic inequalities in depression: A meta-analysis. American Journal of Epidemiology, 157(2), 98-112.

Machida, E. E. R. (2007). Fenômeno bullying e a ética do saber cuidar (Dissertação de mestrado não-publicada). Escola Superior de Teologia, São Leopoldo.

O'Brennan, L. (2009). Examining developmental differences in the social-emotional problems among frequent bullies, victims, and bully/victims. Psychology in the Schools, 46(2), 100-115.

Olweus, D. (1993). Bullying at school: What we know and what we can do. Oxford: Blackwell.

Perren, S., Dooley, J., Shaw, T., \& Cross, D. (2010). Bullying in school and cyberspace: Associations with depressive symptoms in Swiss and Australian adolescents. Child and Adolescent Psychiatry and Mental Health, $4(28), 1-10$.

Pinheiro, F. M. F., \& Williams, L. C. A. (2009). Violência intrafamiliar e envolvimento em bullying no ensino fundamental. Cadernos de Pesquisa, 39(138), 995-1018.

Reppold, C. T., \& Hutz, C. S. (2003). Prevalência de indicadores de depressão entre adolescentes no Rio Grande do Sul. Avaliação Psicológica, 2(2), 175-184.

Sourander, A., Helstelä, L., Helenius, H., \& Piha, J. (2000). Persistence of bullying from childhood to adolescence: A longitudinal 8-year follow-up study. Child Abuse \& Neglect, 24(7), 873-881.

Stelko-Pereira, A. C., Willians, L. C. A., \& Freitas, L. C. (2010). Validade aparente e consistência interna do questionário de investigação de prevalência de violência escolar: estudantes. Revista Avaliação Psicológica, 9(3), 403-411.

Twenge, J. M., \& Nolen-Hoeksema, S. (2002). Age, gender, race, socioeconomic status, and birth cohort differences on the children's depression inventory: A meta-analysis. Journal of Abnormal Psychology, $111(4), 578-588$.

Recebido em: 31/5/2012

Aprovado em: 8/9/2013 
